University of New Hampshire

University of New Hampshire Scholars' Repository

Space Science Center

Institute for the Study of Earth, Oceans, and

Space (EOS)

1997

\title{
Improved COMPTEL 10-30 MeV event selections for point sources from inflight data
}

W Collmar

Max-Planck-Institut für extraterrestriche Physik

U Wessolowski

Max-Planck-Institut für extraterrestriche Physik

V Schonfelder

Max-Planck-Institut für extraterrestrische Physik

G Weidenspointer

Max-Planck-Institut für extraterrestrische Physik

S C. Kappadath

University of New Hampshire - Main Campus

See next page for additional authors

Follow this and additional works at: https://scholars.unh.edu/ssc

Part of the Astrophysics and Astronomy Commons

\section{Recommended Citation}

Improved COMPTEL 10-30 MeV event selections for point sources from inflight data Collmar, W. and Wessolowski, U. and Schönfelder, V. and Weidenspointner, G. and Kappadath, C. and McConnell, M. and Bennett, K., AIP Conference Proceedings, 410, 1587-1591 (1997), DOI:http://dx.doi.org/10.1063/1.54074

This Conference Proceeding is brought to you for free and open access by the Institute for the Study of Earth, Oceans, and Space (EOS) at University of New Hampshire Scholars' Repository. It has been accepted for inclusion in Space Science Center by an authorized administrator of University of New Hampshire Scholars' Repository. For more information, please contact Scholarly.Communication@unh.edu. 
Authors

W Collmar, U Wessolowski, V Schonfelder, G Weidenspointer, S C. Kappadath, Mark L. McConnell, and K Bennett 


\section{AIP $\mid$ proceedings}

Improved COMPTEL 10-30 MeV event selections for point sources from inflight data

W. Collmar, U. Wessolowski, V. Schönfelder, G. Weidenspointner, C. Kappadath,

M. McConnell, and K. Bennett

Citation: AIP Conference Proceedings 410, 1587 (1997); doi: 10.1063/1.54074

View online: http://dx.doi.org/10.1063/1.54074

View Table of Contents:

http://scitation.aip.org/content/aip/proceeding/aipcp/410?ver=pdfcov

Published by the AIP Publishing

Articles you may be interested in

The $100 \mathrm{GeV}$ domain, from ground and from space

AIP Conf. Proc. 558, 519 (2001); 10.1063/1.1370817

Limits on MeV emission from active galaxies measured with COMPTEL

AIP Conf. Proc. 510, 392 (2000); 10.1063/1.1303239

Study of MeV continuum from the Cas A SNR with COMPTEL

AIP Conf. Proc. 510, 60 (2000); 10.1063/1.1303174

The total cosmic diffuse gamma-ray spectrum from 9 to $30 \mathrm{MeV}$ measured with COMPTEL

AIP Conf. Proc. 410, 1218 (1997); 10.1063/1.54105

Earth occultation technique with EGRET calorimeter data above $1 \mathrm{MeV}$

AIP Conf. Proc. 410, 1592 (1997); 10.1063/1.54050 


\title{
Improved COMPTEL 10-30 MeV Event Selections for Point Sources from Inflight Data
}

\author{
W. Collmar ${ }^{1}$, U. Wessolowski ${ }^{1}$, V. Schönfelder ${ }^{1}$, G. \\ Weidenspointner ${ }^{1}$, C. Kappadath ${ }^{2}$, M. McConnell ${ }^{2}, \mathrm{~K}$. \\ Bennett $^{3}$
}

${ }^{1}$ MPI für Extraterrestrische Physik, Germany

${ }^{2}$ University of New Hampshire, USA

${ }^{3}$ Astrophysics Division, ESA/ESTEC, The Netherlands

\begin{abstract}
After several years in orbit the COMPTEL experiment aboard the COMPTON Gamma-Ray Observatory has collected a substantial amount of data from the MeV sky. We have used the inflight event data collected from the Crab, which is the brightest point source at $\mathrm{MeV}$ energies, to optimize our event selections for point sources. For the COMPTEL 10-30 MeV range we have derived a set of improved parameter selections, which leads to a reduction of background events and - at the same time - increases the number of source events, resulting in an obvious improvement in the signal-to-background ratio for point sources. Due to a revised cut on the PSD parameter a background reduction of $\sim 23 \%$ occurs. A narrowing and shifting of the TOF window results in a further slight $(\sim 2 \%)$ reduction of background, however, also in a slight increase $(\sim 5 \%)$ of source events. The revised event cuts improve significances of 'real' point sources in imaging analysis typically by $\sim 1 \sigma$.
\end{abstract}

\section{MOTIVATION}

The COMPTEL experiment [1] aboard the Compton Gamma-Ray Observatory (CGRO) measures $0.75-30 \mathrm{MeV} \gamma$-rays. COMPTEL is the first Compton telescope exploring this difficult energy range from a satellite platform. The sensitivity of COMPTEL - like any other observing instrument - depends on the rate of background events, which is strongly influenced by event selections, i.e. cuts on several recorded event parameters like e.g. the energies on the upper and lower COMPTEL detectors, the time-of-flight (TOF) between the upper and lower detectors, and the pulse-shape discrimination (PSD) to discriminate between $\gamma$-rays and neutrons.

During the first year of the CGRO mission the standard COMPTEL event selections had been derived - neglecting any energy dependence - by using some 
early inflight data from the Crab, which is the brightest point source at $\mathrm{MeV}$ energies. These selections have been modified only slightly over the years. Now, after several years in orbit, much more inflight data on the Crab are available, providing better statistics to investigate the observed COMPTEL event distributions more deeply. This is especially important for the energy bands (e.g. above $10 \mathrm{MeV}$ ), for which the event rate is small and therefore only limited event statistics was available several years ago.

\section{METHOD}

The basic idea of our analysis is to use the Crab source signature in COMPTEL event space as signature for celestial (non-background) events and optimize the parameter cuts on this signature. For the presented analysis we have overlayed 42 days of Crab observations. To derive quantitative results we fitted a simple model to the so-called Angular Resolution Measure (ARM) spectra, which display the angular offset between an event circle and the Crab location (Fig. 1a). The model consists of a first gaussian representing the source (Crab) contribution, and a second gaussian on top of a constant to describe the background contribution. The ARM spectra are generated in a looping procedure for all kinds of combinations of event parameter cuts and are fitted with the model. For each fit we calculated the source and background events as well as the signal-to-background ratio and the source significance for several windows around the best-fit source position. To cross-check our results we applied two supplementory analysis methods: 1 ) background subtraction in event parameters, and 2) visualization of the selected event distributions. Consistency of the different methods was required for final results.

Finally, the modified event selections are checked by applying a complete three-dimensional maximum-likelihood and maximum-entropy data analysis, including standard background generation and the full COMPTEL response.

\section{RESUIJTS}

The analysis has started for all four standard COMPTEL energy ranges (0.75-1 MeV, $1-3 \mathrm{MeV}, 3-10 \mathrm{MeV}, 10-30 \mathrm{MeV}$ ), and the work is still ongoing. Positive results, however, have been derived so far only for the $10-30 \mathrm{MeV}$ range. The event parameters of interest investigated are PSD, TOF, the earth horizon angle $\zeta$, the Compton scatter angle $\bar{\varphi}$, and the so-called cutoff rigidity for cosmic rays. The rigidity dependence on source significances had not been investigated previously, and for PSD only a general restriction was applied to cut out neutron events. Table 1 gives the standard and optimized cuts on these event parameters for the $10-30 \mathrm{MeV}$ range.

The two parameter sets of Table 1 differ mainly in two respects: 1) the narrowing of the PSD window results in a decrease of $\sim 23 \%$ of background 

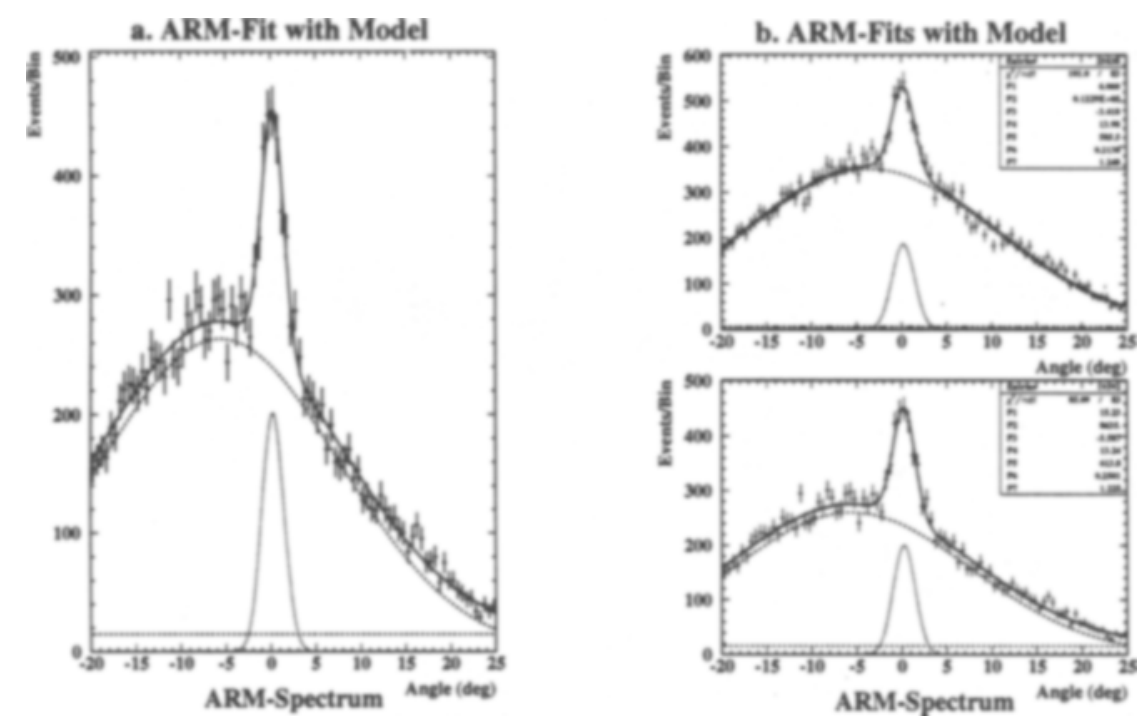

FIGURE 1. (a) Fit of an 10-30 MeV ARM spectrum with our model. The solid line shows the best fit of our model to the data. The dotted line represents the Crab (source) contribution, and the two dashed lines symbolize the individual (constant, gaussian) background components. The total background is the sum of the two dashed lines.

(b) Two selected ARM fits on the same data are shown. The ARM-spectra have been generated by applying the standard (upper panel) and revised (lower panel) selections. The number of events ('entries') decreases significantly while, in contrast, the amplitude for the Crab events (fit parameter 'P5') increases slightly.

events by cutting off the tail of the neutron event distribution but keeping the source events, and 2) the shifting and narrowing of the TOF window results in a further slight decrease $(\sim 2 \%)$ of background events, however, at the same time, in an increase of source events by $\sim 5 \%$ (Fig. 1b). The change of $\zeta$ from $5^{\circ}$ to $0^{\circ}$ is of minor importance, and for the selections on the other two event parameters no improvement could be found. According to our ARM fits the new selections improve the signal-to-background ratio from $38 \%$ to $50 \%$, and the Crab significance from $15.4 \sigma$ to $18.3 \sigma$ for a $\pm 2 \sigma$-window around the best-fit position.

The optimized event cuts yield also improved results in COMPTEL imaging analysis, because the 3-dimensional dataspaces are filled with less background and more source events. Figure 2, as an example, shows two maximumlikelihood images of a three week observation of the Virgo region generated by applying the standard and the revised event cuts. The detection significance of the $\gamma$-ray blazar $3 \mathrm{C} 279$, which showed a strong $\gamma$-ray flare $([2,3])$ during this time period, increases from $\sim 3 \sigma$ to $\sim 4 \sigma$, and the new map appears to be 

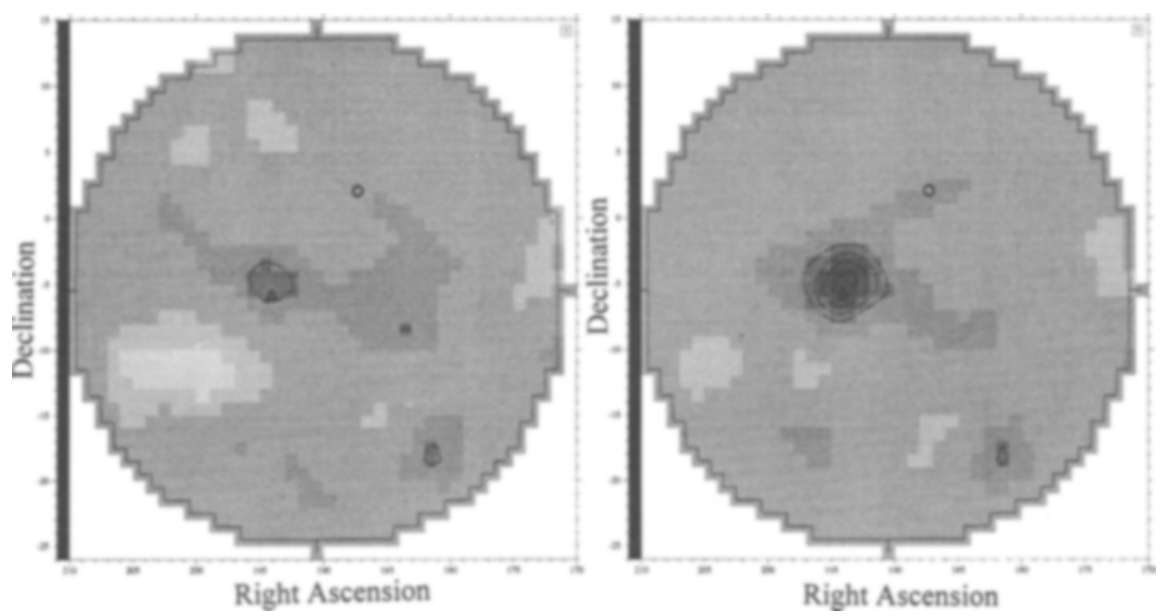

FIGURE 2. Two $10-30 \mathrm{MeV}$ maximum-likelihood images of the Virgo region with an observation time of three weeks (CGRO VPs $511+511.5$ ). The left one was generated with standard selections while the right one was generated with the optimized selections. Both images are displayed on the same greyscale code and contour lining. The contour lines start at a detection significance of $2.5 \sigma$ with a step of $0.5 \sigma$ assuming $\chi_{1}^{2}$-statistics for known sources. The increase in significance $(\sim 3 \sigma$ to $\sim 4 \sigma)$ of the quasar $3 C 279(\triangle)$ is evident.

less noisy. To check whether an improvement for all-sky maps is possible as well, we generated an all-sky maximum-entropy map for the sum of all data up to October '96 (Phase 1 to Cycle 5) using the revised event selections (Fig. 3). This map is considered to be an improvement compared to the standard maps (e.g. [4]), because it is generally 'cleaner' and point sources are visible more clearly, like the blazar PKS 1622-297 $\left(1, \mathrm{~b}: 349^{\circ}, 13^{\circ}\right)$ for example.

\section{SUMMARY}

We have started to revisit the COMPTEL event selection criteria by taking advantage of the increased amount of inflight data. The improved event

TABLE 1. COMPTEL standard and optimized event selections $(10-30 \mathrm{MeV})$ for the different parameters of interest

\begin{tabular}{lccccc}
\hline Selections & $\begin{array}{c}\text { PSD } \\
\text { channel \# }\end{array}$ & $\begin{array}{c}\text { TOF } \\
\text { channel \# }\end{array}$ & $\begin{array}{c}\zeta \\
{\left[{ }^{\circ}\right]}\end{array}$ & $\begin{array}{c}\bar{\varphi} \\
{\left[{ }^{0}\right]}\end{array}$ & Rigidity \\
\hline standard & $0-110$ & $115-130$ & $>5$ & $>4$ & all \\
optimized & $65-85$ & $113-126$ & $>0$ & $>4$ & all \\
\hline
\end{tabular}


CGRO/COMPTEL: All-Sky MEA Map (10-30MeV)

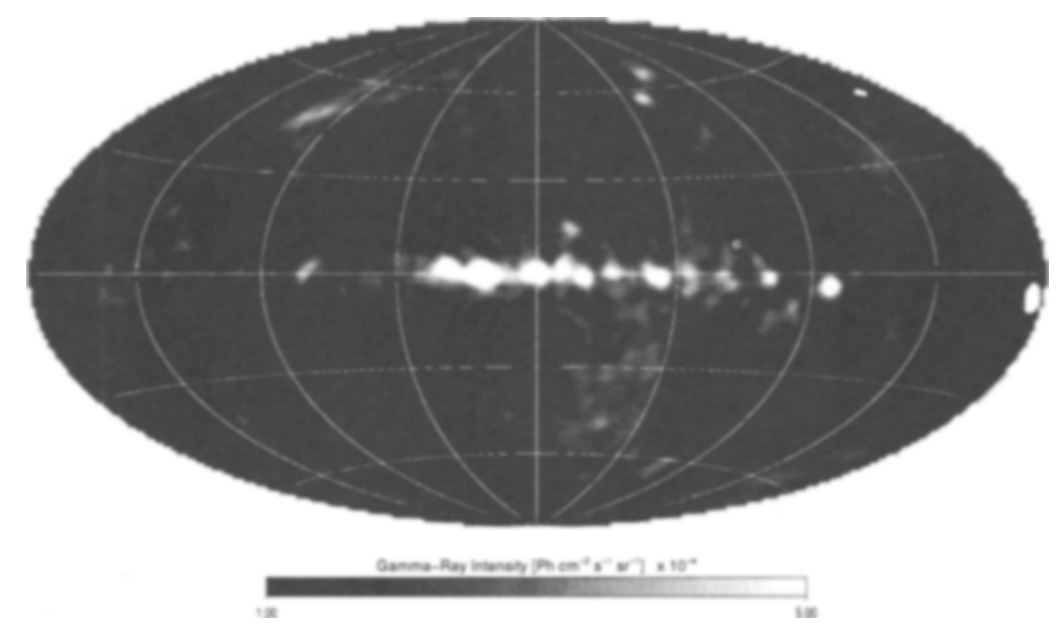

FIGURE 3. COMPTEL $10-30 \mathrm{MeV}$ all-sky maximum-entropy map generated with the optimized event selections and centered at the Galactic Center. Diffuse emission from the galactic plane is obvious. In addition, there are several point sources clearly visible, like e.g. the Crab $\left(1, \mathrm{~b}: 185^{\circ},-6^{\circ}\right)$ and Vela $\left(1, \mathrm{~b}: 264^{\circ}, 3^{\circ}\right)$ pulsars, and the blazars $3 \mathrm{C} 279$ (l,b: $\left.304^{\circ}, 57^{\circ}\right)$, 3C $273\left(\mathrm{l}, \mathrm{b}: 290^{\circ}, 64^{\circ}\right)$, and PKS 1622-297 (l,b: $\left.349^{\circ}, 13^{\circ}\right)$.

statistics offers the opportunity for a detailed study of the event parameter distributions as function of energy, and therefore for improved data selections. First positive results have been achieved for the highest COMPTEL energy range (10-30 MeV), although the optimal selections might not yet be reached. A reduction in background events of $\sim 25 \%$ and - at the same time - an increase of source events by $\sim 5 \%$ has been derived which 'converts' to an improvement of $\sim 1 \sigma$ for 'real' point sources in standard maximum-likelihood imaging analysis. In addition, the maps generally look 'cleaner' because the amplitude of possible artifacts decreases. Investigations of other energy ranges yielded no significant improvements so far, however, work is still in progress. The overall goal is to increase the scientific return from COMPTEL by sensitivity improvements due to optimal event selections.

\section{REFERENCES}

1. Schönfelder V., Aarts H., Bennett K., et al., 1993 ApJS 86, 657

2. Wehrle A., Pian E., Maraschi L., et al., 1997, $A p J$ submitted

3. Collmar W., Schönfelder V., Bloemen H., et al., 1997, these Proceedings

4. Strong A., Diehl R., Oberlack U., et al., 1997, Proc. 2nd Integral Workshop, St. Malo, 1996, ESA SP-382, 533 